\title{
La práctica docente del profesor: La enseñanza de fracciones en un aula de primaria a través de situaciones-problema
}

\section{Teacher practice: The teaching of fractions in a primary classroom through problem situations}

\author{
María Teresa González Astudillo*1 \\ maite@usal.es \\ Ricardo Filipe Marques Portugal** \\ portugal.pt@sapo.pt \\ *Universidad de Salamanca, España \\ **Portugal
}

\section{Resumen:}

En ese documento se trata de identificar los elementos de la práctica del profesor que permiten generar en el aula una discusión sobre el concepto de fracción y que proporcionan oportunidades de aprendizaje a los alumnos. Para ello partimos de dos situaciones-problemas generadas por el maestro, una de ellas correspondiente a la introducción del concepto y la otra a un momento más avanzado del proceso de enseñanza aprendizaje en el que los alumnos tienen que manejar los conceptos aprendidos. La metodología de investigación utilizada es cualitativa, centrada en el estudio de un caso. Los datos se han recogido mediante la grabación de dos sesiones de aula y para su análisis se ha utilizado como marco metodológico el Knowledge Quartet (KQ) haciendo énfasis en las cuatro dimensiones: fundamentación, transformación, conexión y contin-

\begin{abstract}
:
This document seeks to identify the elements in teachers' practice that allow generating a discussion in the classroom about the concept of fraction and provide learning opportunities to students. To this end, we start from two situationsproblems generated by the teacher, one of them is the introduction of the concept and in the other, which corresponds to a later stage of the teaching-learning process, the students have to handle the concepts learned. The research methodology used is qualitative, since it is based on a case study. The data were collected by recording two classroom sessions and the Knowledge Quartet (KQ) was used as a methodological framework, emphasizing its four dimensions: foundation, transformation, connection and contingency. Learning opportunities vary from one task to another. The first one, on being an intro-
\end{abstract}

1 Dirección para correspondencia (correspondence address):

María Teresa González Astudillo. Departamento? Facultad? Universidad? Dirección postal? (España). 
La práctica docente del profesor: La enseñanza de fracciones en un aula de primaria a través de situaciones-problema

María Teresa González Astudillo y Ricardo Filipe Marques Portugal

gencia. Las oportunidades de aprendizaje varían de una tarea a otra. En la primera, al ser una actividad introductoria, están más relacionadas con la conexión mientras que en la segunda tiene más peso la fundamentación y la transformación. Sin embargo, en ambas, la contingencia es un aspecto clave para ahondar en la comprensión de los conceptos.

\section{Palabras clave:}

Resolución de problemas; práctica del profesor; fracción. ductory activity, is more related to connection, while, in the second, foundation and transformation have more weight. However, in both, contingency emerges as a key aspect to delve into the understanding of the concepts.

\section{Key words:}

Problem solving; teacher's practice; fraction.

\section{Résumé:}

Ce document cherche à identifier les éléments de la pratique de l'enseignant qui permettent de générer une discussion en classe sur le concept de fraction et qui offrent des opportunités d'apprentissage aux élèves. Nous commençons donc à partir de deux situations-problèmes générés par le professeur, l'un correspondant à l'introduction du concept et l'autre à un processus d'apprentissage de l'enseignement plus avancé dans lequel les étudiants doivent gérer les concepts appris. La méthodologie de recherche utilisée est qualitative, centrée sur l'étude d'un cas. Les données ont été recueillies en enregistrant deux séances en classe et pour l'analyse, comme cadre méthodologique, le Quartet des connaissances (KQ) a été utilisé et met l'accent sur les quatre dimensions: fondations, transformation, connexion et de contingence. Les possibilités d'apprentissage varient d'une tâche à I'autre. Dans le premier, comme il s'agit d'une activité d'introduction, elles sont plus liées à la connexion tandis que dans la seconde, le fondement et la transformation ont plus de poids. Cependant, dans les deux cas, la contingence est un aspect clé pour approfondir la compréhension des concepts.

\section{Mots clés:}

Résolution de problèmes; pratique de l'enseignant; fraction.

Fecha de recepción: 9-5-2018

Fecha de aceptación: 1-10-2018

\section{Introducción}

Han sido numerosas las investigaciones en las que se describen las dificultades de los alumnos en torno al concepto de fracción. Según Monteiro y Pinto (2006) uno de los obstáculos en la comprensión de este concepto es el énfasis que hacen los profesores en el cálculo algorítmico, las reglas y los procedimientos, relegando a un segundo plano la resolución de problemas. La comprensión de los conceptos matemáticos requiere tener en cuenta su representación y significado. "La representación permite la expresión y uso del objeto. El significado atiende a la interpretación del objeto" (Pecharroman, 2014, p. 112). 
Estos dos son los aspectos en los que nos vamos a centrar en esta investigación.

Ya Kieren (1976) identificó cuatro significados del concepto de fracción: como operador, como razón, como cociente y como medida, mientras que Behr et al. (1992) añadió un nuevo significado: parte-todo. En cuanto a las representaciones, para la enseñanza de las fracciones los maestros usan diversas representaciones como: figuras geométricas, recta numérica, objetos, representación numérica,... aunque no siempre de forma adecuada según el significado de fracción que se está manejando en el aula (Piñón, 1995).

Según Ponte (1995) el profesor tiene que tener un papel esencial en los procesos de cambio curricular, no sólo para interpretarlos correctamente sino también para informar y validar el contenido respectivo. Por ello consideramos que la práctica del profesor es fundamental para comprender en qué se basa su conocimiento y cuáles son los mecanismos que usa y que le ayudan en su vida profesional.

Este estudio, que forma parte de uno más amplio realizado en Portugal, pretende identificar y clasificar los conocimientos matemáticos movilizados por un profesor en la enseñanza de las fracciones en $5^{\circ}$ año de escolaridad, prestando especial atención a aquellos conocimientos que se revelan promotores del desarrollo del concepto. Se trata de un estudio exploratorio centrado en los momentos de discusión con toda la clase. Aquí nos limitamos apenas a la descripción y análisis de dos tareas correspondientes a dos sesiones de clase.

Las investigaciones que se han realizado en torno al conocimiento del profesor tienen aplicaciones tanto en la formación de futuros profesores como para mejorar la práctica docente de los profesores en ejercicio. Esta práctica, como señala Llinares (2013), se nutre tanto de la investigación como de la propia experiencia del docente, de forma que las investigaciones más recientes sobre el conocimiento del profesor se centran en dicha práctica. Tal es el caso de la investigación desarrollada en la Universidad de Michigan por Deborah Ball y colaboradores e identificada con el conocimiento matemático para la enseñanza (MKT).

El objetivo principal que se persigue con el presente estudio es identificar las acciones que realiza el profesor, derivadas de su conocimiento, durante la discusión grupal de la resolución de dos situaciones-problema y cómo estas acciones generan oportunidades de aprendizaje para los alumnos, entendiendo por oportunidad de aprendizaje a un indicador 
La práctica docente del profesor: La enseñanza de fracciones en un aula de primaria a través de situaciones-problema

María Teresa González Astudillo y Ricardo Filipe Marques Portugal

que conecta enseñanza y aprendizaje (Hiebert y Grouws, 2007). Para alcanzar este objetivo se realizará un análisis micro de las dos situaciones sin perder de vista los objetivos que, de forma más general, ha planteado el profesor en sesiones de aula reales.

\section{Marco teórico}

Este trabajo de investigación se encuadra dentro del modelo teórico de Shulman (1986) que propone que el foco de la investigación sobre la enseñanza sea el estudio del conocimiento del profesor acerca de un tópico. Para eso, debe entenderse que toda la actividad educativa tiene como soporte una serie de creencias y de teorías implícitas que forman parte del pensamiento del profesor y que orientan sus ideas sobre el conocimiento así como su enseñanza. Shulman (1986) considera que el conocimiento del profesor está organizado en tres aspectos interrelacionados: el conocimiento del contenido a enseñar (que se denomina Subject Matter Knowledge), el conocimiento didáctico del contenido (que se designa por Pedagogical Content Knowlege) y el conocimiento del currículo (que se designa por Curriculum Knowledge).

En esta investigación interesa el conocimiento del profesor que se muestra en la práctica docente. Esta práctica docente se concretará en torno a dos elementos: las situaciones problema que plantea el profesor y la discusión en gran grupo de la resolución de dichas situaciones. Estas situaciones-problema o tareas son el medio que utiliza el profesor para lograr el aprendizaje de los alumnos. Pueden tener estructuras diferentes, diferente grado de complejidad, involucrar diferentes contextos o presentaciones y pueden dar lugar a una estructuración temporal y organizativa diferentes (Ponte, 2005). Por esto se han escogido dos tareas muy diferentes entre ellas, correspondientes a diferentes momentos de enseñanza, que involucran diferentes aspectos del concepto de fracción y que conllevan el uso de representaciones distintas para poder analizar las oportunidades de aprendizaje.

El otro aspecto que se ha considerado, es el relativo a la discusión en gran grupo, con toda la clase. Este es un elemento clave en esta investigación por cuanto durante las discusiones en grupo se pueden observar las acciones que realiza el profesor a través de registros verbales. Además, la comunicación dialógica, en la que los diferentes participantes 
intervienen desde su rol en el aula permite al profesor "tener en cuenta diferentes puntos de vista... Animando a los estudiantes a participar de una forma exploratoria que ayuda al desarrollo de la comprensión" (Ruthven, Hofmann y Mercer, 2011, p. 81). Para ello, un elemento esencial son las preguntas que realizan tanto el maestro como los alumnos a través de las cuales se puede organizar la discusión, negociando el significado de los conceptos matemáticos o conduciendo a los estudiantes a hacer conexiones entre diferentes conceptos matemáticos (Bishop y Goffree, 1986). En este proceso están involucrados tanto el profesor como los alumnos. El primero por cuanto con sus preguntas incide en los aspectos clave del contenido que se está trabajando en el aula (Sherin, 2002), redirige el pensamiento de los alumnos, muchas veces incluso anticipa las dificultades con las que éstos se encuentran (Ponte y Quaresma, 2016), determina las intervenciones de los alumnos y establece diferentes posibilidades para la resolución de las tareas a partir del trabajo de los alumnos (Stein, Engel, Smith y Hughes, 2008). En cuanto a los alumnos, estas preguntas conducen a desarrollar la competencia relativa a la comunicación matemática, permiten que ajusten el lenguaje matemático, los términos que utilizan, incluso las diferentes representaciones del concepto en cuestión.

Para esta investigación se ha recurrido al modelo Cuarteto del Conocimiento (Knowledge Quartet) de Rowland, Huckstep e Thwaites (2011) que se concreta en un cuadro teórico para la observación, análisis y desarrollo de la práctica de la enseñanza de las Matemáticas. Se seleccionó este marco para el análisis, por cuanto está centrado en el análisis de la práctica y ayuda a identificar las acciones realizadas por el profesor cuando trata de enseñar un tópico matemático para analizar lo que sabe y piensa. Este marco teórico tiene como foco la contribución tanto al conocimiento del contenido matemático como al conocimiento didáctico del contenido del profesor. Se trata de un marco flexible que permite capturar las ideas importantes y encuadrarlas en un número no excesivo de categorías conceptuales que puedan ser eficaces en la investigación. En este modelo se categorizan las situaciones de las aulas de matemática con particular atención al tópico matemático a ser enseñado y al conocimiento matemático que el profesor pone en juego en el aula (Rowland, 2005). El objetivo es lo que el profesor sabe, en qué se fundamenta y cómo identificar las oportunidades para mejorar la enseñanza.

Inicialmente, los investigadores Rowland, Huckstep y Thwaites (2005) 
La práctica docente del profesor: La enseñanza de fracciones en un aula de primaria a través de situaciones-problema

María Teresa González Astudillo y Ricardo Filipe Marques Portugal

a través del análisis de 24 lecciones videograbadas de un futuro profesor cuando enseñaba la sustracción, y mediante un proceso inductivo con una comparación constante, construyeron el marco teórico que organiza el conocimiento del contenido y el conocimiento didáctico del contenido del profesor. Este marco estaba estructurado en cuatro dimensiones y 18 códigos. En un refinamiento posterior generaron un conjunto de 20 categorías que fueron agrupadas por ser de la misma naturaleza o similar en cuatro dimensiones: Fundamentación, transformación, conexión y contingencia (Rowland, Huckstep and Thwaites, 2011).

En la fundamentación se incluyen los conocimientos y la comprensión de la matemática per se y el saber necesario para enseñar matemáticas, así como las creencias sobre la naturaleza de la matemática, las finalidades de la educación matemática y las condiciones sobre las cuales los alumnos aprenderán mejor las matemáticas.

Las otras tres dimensiones se refieren a los modos y contextos en los cuales el conocimiento es aplicado y en la preparación y organización de la enseñanza.

La transformación evidencia que la base del conocimiento para enseñar se distingue por la habilidad del profesor para transformar el conocimiento del contenido que posee en formas que son pedagógicamente fuertes. La conexión incluye la coherencia en la planificación en la enseñanza evidenciada a lo largo del episodio de la clase o de una serie de clases. La contingencia incluye la capacidad de responder del profesor en situaciones de clase que no estaban previstas ni anticipadas.

En el Cuarteto del Conocimiento muchos episodios o momentos pueden ser entendidos en términos de dos o más de las cuatro dimensiones. Por ejemplo, una respuesta a una sugerencia de un alumno (contingencia) puede relacionar ideas anteriores (conexión) o puede necesitar de un conocimiento de la materia a enseñar (fundamentación). Además se puede argumentar que la aplicación del tema en la clase se basa siempre en "lo que el profesor sabe" (fundamentación).

La siguiente tabla (Rowland, Huckstep e Thwaites, 2011) muestra la distribución de las categorías por cada una de las cuatro dimensiones. 
Tabla 1. Categorías del Cuarteto del Conocimiento de Rowland, Huckstep e Thwaites (2011).

\begin{tabular}{ll}
\hline Dimensión & Categorías \\
\hline Fundamentación & $\begin{array}{l}\text { Consciencia de los objetivos, recurre al manual, concentra- } \\
\text { ción en los procedimientos, identificación de errores, ex- } \\
\text { hibición explícita del tema, bases teóricas de la pedagogía, } \\
\text { uso de la terminología matemática }\end{array}$ \\
\hline Transformación & $\begin{array}{l}\text { Selección de ejemplos, selección de representaciones, uso } \\
\text { del material de enseñanza, demostración del profesor (para } \\
\text { enseñar un procedimiento) }\end{array}$ \\
\hline Conexión & $\begin{array}{l}\text { Anticipación de la complejidad, decisiones sobre la secuen- } \\
\text { ciación, conexiones entre procedimientos, conexiones so- } \\
\text { bre conceptos, reconocimiento de la adecuación concep- } \\
\text { tual }\end{array}$ \\
\hline Contingencia & $\begin{array}{l}\text { Desvío del plan de trabajo, responde a las ideas del alumno, } \\
\text { uso de oportunidades, percepción del profesor durante la } \\
\text { clase. }\end{array}$ \\
\hline
\end{tabular}

La adopción de este marco metodológico se realizó por ser de gran utilidad para el estudio del conocimiento del profesor a través de la práctica y por el marcado carácter de la contingencia que permite el análisis de la interacción con el alumno o entre los alumnos y exige un sentido de improvisación que se puede traducir como un momento de aprendizaje para el profesor. Cuando se confronta con la manera de pensar de un alumno, el profesor se ve obligado a una reflexión para reaccionar y tomar una decisión. Dado que el trabajo en el aula se realiza de manera dialógica, se ha considerado este marco como el idóneo para el análisis. Se trata de estudiar al profesor en su ambiente natural, el aula, considerando toda su complejidad. Esto no se logra determinar mediante el uso de otros marcos teóricos.

\section{Metodología y diseño de la investigación}

Este estudio sigue un paradigma cualitativo e interpretativo (Bogdan \& Biklen, 1994) con un estudio de caso. El profesor que participó en el estudio es licenciado en matemáticas y ciencias de un instituto politécnico público de Portugal, imparte las asignaturas de matemáticas y de ciencias desde hace 24 años en un centro de educación primaria. Se considera un profesor interesado e involucrado aunque 
La práctica docente del profesor: La enseñanza de fracciones en un aula de primaria a través de situaciones-problema

María Teresa González Astudillo y Ricardo Filipe Marques Portugal

en los últimos años no ha participado en actividades de formación continua.

Para realizar la investigación se solicitó autorización al director de la escuela que fue el que indicó el interés y la disponibilidad del profesor José (nombre ficticio) para colaborar en el estudio. Por lo tanto, no hubo un criterio específico de selección del docente sino su disponibilidad para participar.

Se realizó una solicitud de autorización a los padres de los alumnos antes de iniciar la recogida de los datos. En total se grabaron en vídeo entre abril y junio de 2012, 17 clases de un grupo de $5^{\circ}$ grado en las que se abordó el concepto de fracción y que posteriormente fueron transcritas en su totalidad. Además de la grabación, el investigador realizó una observación que plasmó en un diario a modo de registro. Antes del inicio del estudio se realizó una entrevista semiestructurada al profesor sobre su trayectoria profesional e inquietudes.

Los datos que se han considerado para este artículo han sido los relativos a la primera entrevista del profesor, el diario de observación del investigador y la grabación en audio y vídeo de las dos sesiones de aula mencionadas.

El análisis se centró en la práctica lectiva del profesor sin hacer referencia a los alumnos. Se llevó a cabo después de la transcripción de todas las sesiones y de la entrevista. Se analizaron por separado cada una de las tareas realizadas en el aula mediante un análisis de contenido que permitió el paso de los datos brutos a los datos organizados sin desviarse del material, pero haciendo evidentes aspectos invisibles inicialmente. Las tareas se dividieron en segmentos a los que se fue asociando una o más de las dimensiones del modelo KQ y que se fueron categorizando por los investigadores procurando la triangulación de los investigadores.

\section{Resultados y discusión}

A continuación, se presenta el análisis de dos de las tareas analizadas. La primera de ellas corresponde a una actividad introductoria en la que el profesor plantea el concepto de fracción en un contexto de medida relativo a la comparación de áreas de figuras planas. La segunda es una tarea planteada a los alumnos hacia la mitad de toda la programación relativa a la suma de fracciones, por tanto, los alumnos ya poseen algu- 
nos conocimientos de este concepto y durante esta sesión el profesor va a procurar integrarlos. En las transcripciones no se ha hecho distinción de los alumnos puesto que el análisis está centrado en el profesor. Se presentan estos resultados manteniendo la cronología para comprender mejor el proceso que se ha seguido en cada tarea e indicando la dimensión/categoría que se pone de manifiesto en cada momento. En los extractos seleccionados a continuación, se indica la intervención del profesor mediante una $\mathrm{P}$ y la de un alumno con una A. Se ha procurado mantener la opción dialógica utilizada en el aula.

\section{Introduciendo el concepto de fracción: La fracción como medida}

La situación que se propone es la siguiente: El profesor distribuye a los alumnos un tangram. Las figuras del tangram van a servir de comparación unas con otras para establecer la medida de su área.

El profesor comienza la clase repartiendo a cada uno de los alumnos un tangram (transformación-uso de material) y plantea inicialmente una actividad exploratoria para que se vayan familiarizando con el material de forma que vayan distinguiendo las figuras que lo conforman y las características que poseen, además de diferenciarlas. Les pide a los alumnos que realicen construcciones con las piezas al mismo tiempo que van discutiendo en gran grupo diferentes cuestiones que plantea el profesor. Esta fase de familiarización con el material didáctico permite al profesor repasar y afianzar otros conceptos matemáticos, en este caso geométricos, como es la clasificación de las formas planas (conexión-entre conceptos). En este caso el aspecto central que centra la discusión son las características geométricas de las figuras que conforman el material, lo que lleva a clasificarlas (fundamentación).

P: Una pieza como esta ¿Cómo se llama?

A: Triángulo

P: Triángulo. Clasifícame este triángulo en cuanto al ángulo. Nosotros no hemos dado bien esto, pero bueno. En cuanto a los ángulos ¿Cómo es este de aquí?

A: Recto

P: ¿Cómo se llama este triángulo? ¿Quién ayuda a Ana? El triángulo que tiene 
La práctica docente del profesor: La enseñanza de fracciones en un aula de primaria a través de situaciones-problema

María Teresa González Astudillo y Ricardo Filipe Marques Portugal

un ángulo recto. Nosotros todavía no lo hemos dado pero esto son cosas que... ¿quién lo sabe?

A: Rectángulo

P: Triángulo rectángulo. Muy bien. ¿En cuanto a la longitud de los lados? Esto ya salió en vuestros test ¿Este lado y este?

A: Son iguales

P: Entonces ¿cómo se llama un triángulo que tiene dos lados iguales, con la misma longitud?
A: Equilátero
$P:$ No ¿cómo se llama?
A: Isósceles

La discusión anterior ha permitido al profesor revisar la clasificación de los triángulos según sus lados y sus ángulos (fundamentación-exhibición explícita del tema), recordar los términos correspondientes a los diferentes tipos de triángulos y ajustar estos términos ante la confusión de uno de los alumnos (triángulo isósceles). A partir de aquí, se identifican otros tipos de figuras entre las piezas del tangram: triángulo, cuadrado, romboide, así como el número de figuras que hay de cada tipo. Hay una figura que causa algún problema lo que lleva a que el profesor aproveche la cuestión para que los alumnos utilicen el término adecuado y que lo relacionen con las características que lo definen (fundamentación-uso de la terminología adecuada).

P: A esta figura no le hemos dado nombre todavía. El nombre de esta figura lo vamos a ver hoy pero no lo vamos a dar hasta $8^{\circ}$ ¿Alguien sabe su nombre?

A: Paralelepípedo

P: ¿Paralelepípedo? ¿qué es un paralelepípedo? Es un sólido

A: Paralelogramo

$P$ : Tiene un nombre más específico que el de paralelogramo. Se llama romboide, pero van a ver que es un paralelogramo. Entonces ¿Por qué es un paralelogramo? La propia palabra lo dice ¿qué dice la palabra paralelogramo?

A: Es paralelo

P: Dice que los lados son paralelos, Los lados opuestos son paralelos

Una vez reconocidas las figuras se plantea una comparación entre ellas para determinar la medida de las áreas de unas en función de las otras (noción de medida del área por comparación). La comparación en 
este caso se está haciendo por superposición de las figuras. Con ello se introduce el concepto de fracción con el significado de medida (fundamentación, transformación-enseñar un procedimiento).

P: Vamos a comparar el área de los dos triángulos pequeños con la del cuadrado.

\section{A: Es igual}

$P:$ ¿Con los dos triángulos pequeños consiguen construir el cuadrado?

A: Si

P: Entonces comparen el área de los dos triángulos pequeños con la del paralelogramo

A: Aquí también da, pienso yo

$P$ : Comparen

A: Es igual

(...)

P: Ahora comparen el área del cuadrado con el área del paralelogramo

A: Es igual

El profesor ha propiciado una situación en la que los alumnos han utilizado una relación de igualdad de áreas de las figuras (transformación y conexión). La comparación de esas áreas se ha hecho por superposición y finalmente se ha conseguido utilizar la propiedad transitiva de la relación para establecer que el paralelogramo y el cuadrado tienen la misma área.

Se introduce en este momento como unidad de medida el área del triángulo pequeño (fundamentación-unidad de medida no convencional) para establecer la medida de las áreas del resto de las figuras.

P: Cuando utilizamos como unidad de medida el triángulo, entonces díganme ¿cuál es el área del cuadrado si la unidad de medida es el triángulo?

A: ¿El triángulo pequeño?

P: Si. ¿Cuál es el área del cuadrado?

A: Dos triángulos

P: Dos, dos unidades, dos triángulos. ¿Y cuál es el área del paralelogramo si utilizamos como unidad de área el triángulo pequeño?

A: Dos triángulos

P: Entonces ¿qué concluimos? Si esto tiene área... ¿cuál es el área de este?

A: Dos 
La práctica docente del profesor: La enseñanza de fracciones en un aula de primaria a través de situaciones-problema

María Teresa González Astudillo y Ricardo Filipe Marques Portugal

$P$ : Entonces ¿ $Y$ este tiene área?

A: Dos

P: Entonces ¿los dos tienen o no el mismo área?

A: Sí

$P:$ ¿Y cómo se llaman las figuras que tienen el mismo área?

A: Equivalentes

Hace lo mismo para el triángulo mediano de lo que los alumnos concluyen que también tiene área 2 y llegan a la conclusión de que hay tres figuras que tienen la misma área: el triángulo mediano, el cuadrado y el paralelogramo. Todo esto lo hacen de forma manipulativa, experimentando y comprobando con las piezas para determinar las áreas de cada una de las figuras.

Hacen lo mismo para el triángulo grande y llegan a la conclusión de que su área se corresponde con 4 triángulos pequeños. Como no tienen cuatro triángulos pequeños el profesor les dice que lo hagan utilizando los dos triángulos pequeños y el cuadrado. Insiste con ellos en que deben encajar las piezas para comprobar la relación entre las áreas. Algunos alumnos no consiguen colocar bien las piezas lo que permite percibir otro momento de contingencia en la sesión de clase, hasta que el alumno se da cuenta de cómo debe colocar las piezas para obtener la figura adecuada.

P: Experimenta. Así no da. Tienes que poner los triángulos de otra forma. Si no da de una manera ... Si intentas colocar el cuadrado en primer lugar y luego experimentas

A: Ah Ya da.

P: ¿Ya da? Entonces, díganme una cosa. ¿Cuál es el área del triángulo grande si la unidad de área fuera el triángulo pequeño?

A: 4

A1: Es el doble

Una vez que están establecidas las relaciones entre las piezas y que se han medido utilizando como unidad el triángulo pequeño el profesor les pide que completen una tabla (tranformación-uso de representaciones). Primeramente, les explica la estructura de la tabla. Las figuras que están en la primera fila se van a utilizar como unidad de área para medir el área de las figuras (conexión-comparación entre áreas y relación con 
las fracciones) que están en la primera columna (fundamentación-explicación de un procedimiento).

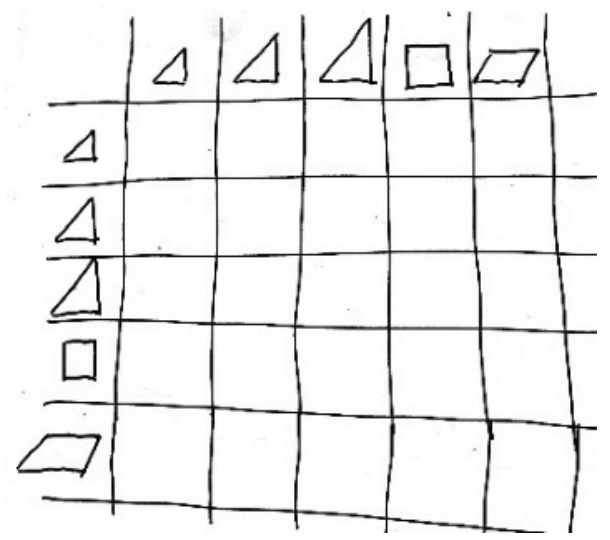

Figura 1. Comparación de áreas variando la unidad.

Para completarla se pide a los alumnos que trabajen en parejas y establezcan las relaciones entre las diferentes piezas del tangram para lo que les da un margen de tiempo. Se trata de que determinen las áreas de las figuras cuando se va cambiando la unidad de medida y se van utilizando para tal fin las diferentes piezas del trangram. Las fracciones que así se obtienen son fracciones básicas, propias, con numerador igual a uno (conexión- secuenciación). Para que los alumnos entiendan cómo se puede establecer la relación entre las piezas el profesor les ayuda con un ejemplo. Ante las dificultades de los alumnos para determinar la medi$\mathrm{da}$, trata de hacerlos razonar para que entiendan la tarea (contingencia). Cuando algún alumno no encuentra la respuesta correcta, el profesor le hace preguntas diferentes hasta que la obtiene de forma razonada. Esta parte de la actividad constituyó un reto para los alumnos al tener que utilizar como unidades de área figuras más grandes que aquellas que querían medir.

P: ... Atención voy a hacer la pregunta de dos formas. Si la unidad de área fuera el triángulo pequeño ¿cuál es el área de esta? [está mostrando el cuadrado]

A: Dos triángulos

P: Si la unidad de área fuera esta [cuadrado] ¿Cuál es el área del triángulo pequeño? 
La práctica docente del profesor: La enseñanza de fracciones en un aula de primaria a través de situaciones-problema

María Teresa González Astudillo y Ricardo Filipe Marques Portugal

A: Dos

A1: Dos

A2: La mitad

P: Pedro. Hay que razonar. Dije hace un momento que ésta es la figura que tú tienes. Ésta es la unidad de área ¿cuántas veces cabe el triángulo pequeño aquí?

A: Dos

P: Entonces tú dices que el área de esta es 2. Vamos al contrario. Esto pasa a ser la unidad de área [el triángulo pequeño]. Tú tienes esto ¿Cuánto es esto en relación con eso?

A: 0,5

P: ¿Cuántas veces cabe esto aquí? Pedro ¿Cuántas veces cabe esto aquí?

A: Una

$P:$ ¿Cabe una? Entonces ¿tienen la misma área?

A: No

P: Cabría una vez si tuviera el mismo...

A: Área

P: Pero no tienen la misma área. Entonces, si esta figura tiene el doble área que esta ¿Qué pasa con esta en relación con esta? Esta tiene...

A: La mitad.

Una vez que los alumnos se han aclarado, el profesor discute con ellos las distintas formas de representación de esa cantidad [transformación-uso de diferentes representaciones]. Pregunta a los alumnos qué opciones tienen y surgen como posibilidades $1 / 2,0,5$ y 50\%. Luego les pregunta por la expresión oral de esa cantidad. El profesor insiste mucho en ese último aspecto, la lectura de los números decimales (fundamentación-terminología matemática), para que los alumnos hagan alusión a las diferentes posiciones decimales (cinco décimas).

P: ¿Cómo lo expresaríamos con el lenguaje oral?

A: Un medio

P: Acepto que lo pongan de dos maneras ¿Cómo se lee 0,5?

A1: Cinco décimas

Una vez aclarada la tarea, los alumnos la resuelven mientras el profesor revisa los avances que van teniendo y responde a las dudas que surgen (contingencia). Les insiste en que tienen que completar la tabla con fracciones, no con números decimales. 
La práctica docente del profesor: La enseñanza de fracciones en un aula de primaria a través de situaciones-problema María Teresa González Astudillo y Ricardo Filipe Marques Portugal
A: Profesor
P: Dime

A: Este hace un triángulo pequeño, este vale dos, y este vale cuatro, es el cuadrúple

P: Exactamente, Muy bien. Uno es el cuadrúple del otro y el otro ¿Cómo se dice si uno es el cuádruple del otro como es el otro?

\section{A: Cuarta parte}

Después de un rato que los alumnos están trabajando en la tabla surge una nueva discusión. Los alumnos se dan cuenta que algunas figuras tienen la misma área y que pueden utilizar esa relación para rellenar más fácilmente la tabla. Aprovechan esta discusión para completar la primera columna de la tabla.

\section{A: Profesor \\ P: Dime}

A: Si este de aquí son dos pequeños y este también, entonces es igual

P: Nosotros ya habíamos llegado a la conclusión. Atiendan todos. Antonio está preguntando una cuestión. Paren cinco segundos. Nosotros hemos llegado a una conclusión hace un rato. Había tres piezas que tenían la misma área. ¿Cómo las llamábamos?
A: Equivalentes
P: Eran equivalentes. Nosotros no las llamábamos equivalentes. Utilizamos otro nombre ¿Cómo las llamábamos?
A: Congruentes
$P$ : Si estos tres tienen la misma área, piensen lo que va a pasar en las columnas
A: Profesor, si este de aquí es igual a este entonces es una vez.
P: Obligatoriamente. Antonio, obligatoriamente (.... ) Todos nos vamos a fijar sólo en la primera columna ¿Cuál es el área de este?

A: Uno

P: ¿Por qué? Porque son iguales ¿Cuál es el área del triángulo mediano?

A: Dos

$P:$ ¿Cuál es el área del triángulo grande?
A: Cuatro
$P:$ ¿Cuál es el área del cuadrado?

A: Dos

$P:$ ¿Cuál es el área del paralelogramo?

A: Dos 
La práctica docente del profesor: La enseñanza de fracciones en un aula de primaria a través de situaciones-problema

María Teresa González Astudillo y Ricardo Filipe Marques Portugal

P: ¿Todos están de acuerdo?

A: Si (en coro)

Les deja otro rato que razonen y completen la tabla y luego realizan una puesta en común de los resultados de la segunda columna. Selecciona los alumnos que quiere que vayan contestando a las preguntas que él va haciendo de forma que todos estén involucrados en la tarea y para comprobar que la han entendido. Y siguen con el resto de las columnas.

P: Entonces avanzamos a la segunda columna

A: Yo ya la hice

P: Muy bien. Vamos avanzando (...) Ahora la unidad de área es el triángulo mediano. En la segunda columna ¿Cuál es el área del triángulo pequeño? Beatriz ¿Cuál es el área del triángulo pequeño en relación con el triángulo mediano?

A: Mitad

P: Podemos escribir entonces uno barra 2. ¿cuál es el área del triángulo mediano?

A: Uno

P: Uno porque tiene la misma área ¿y cuál es el área de este?

A: Dos triángulo medianos

$P:$ ¿Y cuál es el área del cuadrado? Beatriz, hace un momento tu llegaste a la conclusión de que estas tres eran...

A: Iguales

P: Equivalentes. Entonces todas tienen la misma área. El área de este es igual al área de este ¿Cuál es la relación?

A: Uno

El profesor repasa con los alumnos las columnas correspondientes al cuadrado y al paralelogramo como unidades de área de la misma forma que ha hecho con los otros. En un determinado momento, el profesor les dice que van a hacerlo de otra manera (contingencia-uso de oportunidades). Esto constituye otra oportunidad de aprendizaje porque los alumnos tienen que utilizar la relación de igualdad entre las áreas de las figuras para rellenar la tabla.

P: Hay tres figuras que son equivalentes, ¿cierto? Pedro ¿Cuáles son?

A: Era el triángulo mediano 
La práctica docente del profesor: La enseñanza de fracciones en un aula de primaria a través de situaciones-problema María Teresa González Astudillo y Ricardo Filipe Marques Portugal

P: El triángulo mediano. Lo voy a buscar aquí. Vamos a razonar de otra manera ahora para ver si pensamos más sobre lo que estamos haciendo. El triángulo mediano. ¿Otra?

A: El pequeño

$P$ : Equivalentes, que tengan el mismo área.

A: Ah. El cuadrado

P: El cuadrado. ¿Cuál era el otro que tenía la misma área?

A: Era, el.. como se llama

P: Era el paralelo...

A: El paralelogramo

P: Entonces va a suceder una cosa. Vamos a pensar un poco. Piensen, con el triángulo dio esta columna.

A: Con los otros es todo igual.

P: Entonces la relación entre las columnas es la misma, es evidente. Entonces esta columna tiene que tener la misma relación que esta columna y tiene que tener la misma relación que esta columna lla del cuadrado, el triángulo mediano y el paralelogramo], porque tienen que tener la misma área ¿lo ven? Por tanto, a veces pueden experimentar con las figuras, pero podemos pensar: si tienen el mismo área, la unidad de área es la misma, entonces la relación tiene que ser la misma.

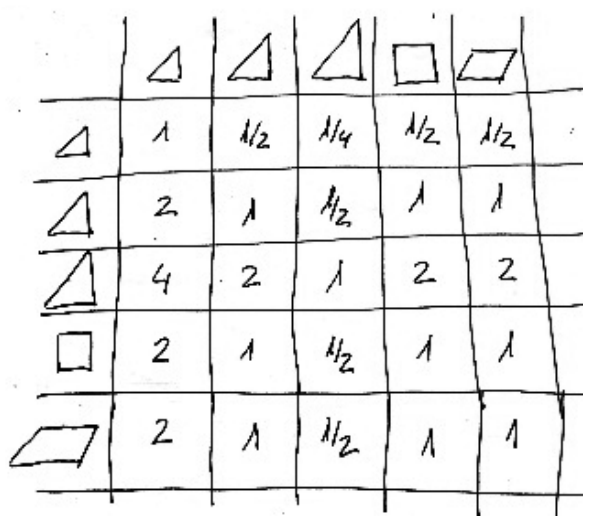

Figura 2. Resultado final de la tarea.

Finalmente consiguen completar toda la tabla como se puede ver en la figura anterior.

Al ser esta una actividad introductoria, el profesor ha recurrido a un material manipulativo, a conocimientos conocidos por los alumnos como son las formas geométricas básicas y el concepto de área. Esto 
La práctica docente del profesor: La enseñanza de fracciones en un aula de primaria a través de situaciones-problema

María Teresa González Astudillo y Ricardo Filipe Marques Portugal

le ha permitido enlazar conocimientos que dominan con otros que son nuevos. La tarea ha sido un reto para los alumnos y, en ciertas ocasiones, el profesor se ha encontrado con momentos de contingencia que se han convertido en oportunidades de aprendizaje para realizar conexiones entre conceptos (figuras con la misma área), introducir conceptos nuevos (romboide) o trabajar cuestiones de índole espacial (rellenar un triángulo con otros dos triángulos y un cuadrado).

\section{Suma de fracciones: diferentes métodos, diferentes representaciones}

La otra tarea es un problema sobre suma de fracciones que se plantea en la sesión novena. Es un problema sencillo pero que da lugar a diferentes formas de abordarlo y el profesor aprovecha cada una de ellas como oportunidades de aprendizaje. El problema es el siguiente: Mariana compró una pizza. Comió un cuarto en la comida y cinco octavos a la cena. ¿Qué parte de la pizza comió Mariana?

Lo primero que hacen en el aula es una lectura comprensiva en gran grupo intentando, entre todos, desentrañar el significado de las fracciones que se mencionan en el problema (fundamentación-conocimiento de las dificultades de los alumnos y de la metodología de enseñanza). Luego les deja un rato para que resuelvan el problema individualmente. Mientras tanto él va comprobando los procesos que van siguiendo los alumnos y los resultados a los que Ilegan. En esa comprobación se da cuenta de que los alumnos utilizan tres procedimientos diferentes (contingencia-uso de oportunidades) lo que aprovecha para organizar la puesta en común y que los alumnos sean conscientes de esos procedimientos, así como que perseveren en la resolución de problemas y se den cuenta que siempre pueden encontrar un método más o menos sencillo para abordarlos.

Vamos a describir cada uno de esos procedimientos. Algunos alumnos realizan una resolución gráfica (transformación-selección representación). El profesor les interroga acerca del procedimiento que han usado para que sepan razonar las decisiones que han tomado y justifiquen por qué lo han hecho de esa forma. El hecho de tener dos fracciones con diferentes denominadores constituye una dificultad a la hora de elegir las partes en las que dividir la unidad. El profesor aprovecha esta opor- 
tunidad para que ellos razonen por qué han elegido dividir la pizza en ocho partes.

P: A ver, Julia

A: Yo dividí la pizza en 8 partes.

P: Ocho partes ¿Por qué?

A: Porque una de las fracciones representa cinco octavos.

$P:$ ¿Y la primera?

A: La primera, un cuarto. Pero como un cuarto,... cinco octavos, es el doble.

$P$ : Entonces, estás diciendo que dividir en 8 partes es el doble. Estás diciendo que como 8 es divisible por 4, podemos dividir en 8.

A: Después pinté un cuarto. Un cuarto son dos octavos.

P: Un cuarto son dos octavos. Son dos trozos ¿no? ¿Y a la cena?

A: Comió cinco trozos.

P: Entonces ¿cuánto comió en total?

A: Siete octavos.

Otros alumnos resolvieron el problema de una forma diferente, sumando las fracciones numéricamente. Para ello utilizan la noción de fracciones equivalentes y el algoritmo de la suma de fracciones (fundamentación-conocimiento del tema). El profesor aprovecha el procedimiento de solución (fundamentación-concentración en los procedimientos) para comprobar si los alumnos han comprendido estos dos conceptos mediante las preguntas que les va haciendo durante la puesta en común.

P: Explica esas cuentas.

A: Pasé un cuarto a octavos.

$P$ : Entonces pensaste en fracciones equivalentes con el mismo denominador.

Pasaste un cuarto a dos octavos para tener el mismo denominador. $Y$ como pasaste un cuarto para dos octavos, ahora puedes hacer...

A: Después sumé dos octavos y cinco octavos

P: y ¿cómo se suman fracciones con el mismo denominador? ¿Por qué dices que dio siete octavos y no dijiste siete dieciseisavos?

A: Porque el denominador no cambia

$P:$ ¿Por qué cuando sumamos se suman los numeradores y no se suman los denominadores? ¿Por qué Julia?

A: Porque la pizza está dividida en 8 partes 
La práctica docente del profesor: La enseñanza de fracciones en un aula de primaria a través de situaciones-problema

María Teresa González Astudillo y Ricardo Filipe Marques Portugal

En el tercer procedimiento los alumnos transforman las fracciones en números decimales (transformación-selección de representaciones). Esto lo aprovecha el profesor para recordar la lectura de números decimales finitos y para hacer cálculo mental (fundamentación-uso de terminología, concentración en los procedimientos).

A: Yo transformé en un número decimal

P: Entonces, dinos cómo obtuviste el número decimal

A: Dividí uno entre cuatro.

$P$ : ¿Qué dio?

A: Veinticinco centésimas

P: Después dividiste cinco entre ocho.

A: 625 milésimas.

P: Muy bien ¿Y ahora?

A: Sumamos

P: Sumamos ¿Y cuánto dio la suma?

A: 875 milésimas.

Un alumno comenta que no entiende muy bien este procedimiento porque el resultado no es el mismo que el de los anteriores (contingencia). Esta situación la aprovecha el profesor para revisar con ellos la transformación de números decimales finitos en fracciones y la simplificación de fracciones, así como el criterio de divisibilidad por 5 (fundamentación-conocimiento de la materia). Así se complementa lo realizado ya que en el extracto anterior en el que se ha transformado una fracción en número decimal.

P: Vamos a hacer de otra manera. No está prevista, pero vamos a confirmar que siete octavos es lo mismo que 875 milésimas. Escriban 875 milésimas en forma de fracción.

A: 875

$P:$ : Sobre?

A: 1000

P: Y ahora vamos a escribir la fracción irreducible. ¿Por cuánto podemos dividir?

A: Por 5

P: ¿Por qué? Porque ambos terminan en... el guarismo de las unidades es 5.

Entonces los números terminados 
A: En 0 o en 5 son divisibles por 5

P: Vamos a dividir por 5 los dos. Vamos a simplificar esto. ¿1000 entre 5?

A: 200

P: 875 entre 5. La división por un divisor que sólo tiene una cifra es fácil.

A: 175

$P:$ ¿Y ahora qué hago?

A: Continúo dividiendo

(...)

P: Entonces ¿35 dividido entre 5?

A: 7

$P: ¿ 40$ entre $5 ?$

A: 8

P: Muy bien. Esto es posible porque tenemos decimales finitos

Esta tarea ha permitido que el profesor muestre diferentes formas de resolución utilizadas por los alumnos. Se trata de un problema que los alumnos han resuelto utilizando tres formas de representación distintas: representación gráfica, fraccionaria, decimal. Durante la discusión el profesor ha aprovechado diferentes oportunidades para repasar con ellos: los algoritmos de suma de fracciones, la transformación de fracción a número decimal y viceversa, el cálculo mental o la equivalencia de fracciones.

\section{Conclusiones}

El análisis de la práctica del profesor ha permitido comprobar las oportunidades de aprendizaje que surgen en la realización de tareas sobre fracciones que pueden llegar a ser un reto para los alumnos. Esto se ha realizado analizando la discusión en gran grupo posterior a la resolución de las tareas, en un ambiente dialógico en el que tanto el profesor como los alumnos están habituados a plantear y responder preguntas. El profesor para indagar acerca de la comprensión lograda por los alumnos y éstos para plantear dificultades, dudas o incluso otras formas de abordar las soluciones. En este caso se han presentado sólo dos tareas que pueden ser una muestra del trabajo realizado en el aula. Corresponden a diferentes momentos de enseñanza, los objetivos de cada una de ellas son diferentes, la primera se apoya en el uso de material manipulativo 
La práctica docente del profesor: La enseñanza de fracciones en un aula de primaria a través de situaciones-problema

María Teresa González Astudillo y Ricardo Filipe Marques Portugal

mientras que la segunda requiere una solución individual previa. Aunque las tareas están pensadas para que los alumnos puedan resolverlas con sus conocimientos, cada una de ellas presenta algún aspecto que resulta cierto reto para los alumnos.

Las acciones que realiza el profesor están mediadas por su conocimiento que se ha puesto de manifiesto por medio de las dimensiones y categorías del KQ. Con la primera tarea el profesor fundamentalmente procura realizar conexiones con otros conceptos y procedimientos a través del material didáctico como: clasificación de figuras geométricas, nombres de las figuras, medida de áreas, comparación de áreas, relación de igual de áreas, propiedad transitiva. Con la segunda la dimensión clave es la fundamentación a través de la cual se ponen en juego conceptos y procedimientos relativos a la equivalencia de fracciones, su suma, la transformación de fracción en decimal y viceversa, los criterios de divisibilidad o el cálculo mental. En ambas ha sido esencial la transformación que ha permitido el trabajo con diferentes representaciones: representaciones gráficas, expresiones decimales, términos para leer las expresiones numéricas.

Esto se ha logrado por la forma en la que el profesor tiene de organizar la comunicación en el aula. Aunque él es quien dirige la discusión, mediante sus preguntas trata que los alumnos alcancen la comprensión, que entiendan los procedimientos, que justifiquen sus decisiones y que razonen los resultados. Además, los alumnos, en todo momento plantean preguntas acerca de lo que supone una duda (contingencia) y el profesor las aprovecha como oportunidades para afianzar los conceptos, relacionarlos con otros o incluso introducir aspectos novedosos que no estaban ni siquiera planificados. La contingencia determina la práctica del profesor en aspectos como: la lectura comprensiva de los enunciados, realización conjunta de alguna parte de la tarea, control de las diferentes soluciones, utilización de razonamientos cuando los alumnos cometen errores, entre otros.

En resumen, se ha comprobado cómo tanto las situaciones como la comunicación dialógica contribuyen a crear oportunidades de aprendizaje en el aula. Para ello, es claro que son esenciales tanto el conocimiento del profesor sobre la materia, como sobre enseñanza del concepto y su experiencia previa. 
La práctica docente del profesor: La enseñanza de fracciones en un aula de primaria a través de situaciones-problema María Teresa González Astudillo y Ricardo Filipe Marques Portugal

\section{Referencias}

Behr, M., Harel, G., Post, T. \& Lesh, R. (1992). Rational number, ratio and proportion. En D. A. Grouws (Ed.) Handbook of Research on Mathematics Teaching and Learning (pp. 233-296). New York: MacMillan Publishing Company.

Bishop, A., \& Goffree, F. (1986). Classroom organization and dynamics. In B. Christiansen, A. G. Howson, \& M. Otte (Eds.), Perspectives on mathematics education (pp. 309-365). Dordrecht: Reidel.

Bogdan, R., \& Biklen, S. K. (1994). Investigação qualitativa em educação: Uma introdução à teoria e aos métodos. Porto: Porto Editora.

Hiebert, J. \& Grouws, D. (2007) The effects of classroom mathematics teaching on students' learning. En F. Lester (Ed.). Handbook of Research on Mathematics Teaching and Learning (pp. 371-404). NCTM: Information Age Publishing.

Kieren, T. E. (1976). On the mathematical, cognitive and instructional foundations of rational numbers. En R. Lesh (Ed.), Number and measurement: Papers from a research workshop (pp. 101-144). Columbus, OH: ERIC/SMEAC.

Llinares, S. (2013). Professional noticing: A component of mathematics teacher's professional practice. Sisyphus, 1(3), 76-93.

Monteiro, C. \& Pinto, H. (2006). A aprendizagem dos números racionais. Quadrante, 14(1), 89-108.

Pecharroman, C. (2014). El aprendizaje y la comprensión de los conceptos matemáticos desde una perspectiva ontológica. Educación matemática, 26(2), 111-133.

Piñón, M. (1995). Las fracciones en la escuela. Pedagogía, 10(5), 58-65.

Ponte, J. P. (1995). Perspectivas de desenvolvimento profissional de professores de Matemática. In J. P. Ponte, C. Monteiro, M. Maia, L. Serrazina, \& C. Loureiro (Eds.), Desenvolvimento profissional de professores de Matemática: Que formação? (pp. 193-211). Lisboa: SEM-SPCE

Ponte, J. P. (2005). Gestão curricular em Matemática. In GTI (Ed.), O professor e o desenvolvimento curricular (pp. 11-34). Lisboa: APM.

Ponte, J.O. y Quaresma, M. (2016) Teachers' professional practice conducting mathematical discussion. Educational Studies in mathematics, 93(1), 51-66.

Rowland, T., Huckstep, P. y Thwaites, A. (2005) Elementary teachers' mathematics knowledge: the knowledge Quartet and the case of Naomi. Journal of Mathematics Teacher Education, 8, 255-281.

Rowland, T. (2005). The Knowledge Quartet: a tool for developing mathematics teaching. Proceedings of the Forth Mediterranean Conference on Mathematics Education, 28, 29, 30. January, Palermo, pp.69-81.

Rowland, T., Huckstep, P. e Thwaites, A. (2011) Secondary mathematics teachers' content knowledge: the case of Heidi. Paper given at CERME7, pp. 2817-2837. Rzeszów, Poland.

Ruthven, K., Hofmann, R., \& Mercer, N. (2011). A dialogic approach to plenary problem synthesis. In B. Ubuz (Ed.), Proceedings of the 35th Conference of the International Group for the Psychology of Mathematics Education (Vol. 4, pp. 81-88). Ankara, Turkey: PME. 
La práctica docente del profesor: La enseñanza de fracciones en un aula de primaria a través de situaciones-problema

María Teresa González Astudillo y Ricardo Filipe Marques Portugal

Sherin, M. G. (2002). A balancing act: Developing a discourse community in the mathematics classroom. Journal of Mathematics Teacher Education, 5, 205-233.

Shulman, L. S. (1986). Those who understand: knowledge growth in teaching. Educational Research, 15(2), 4-14.

Stein, M. K., Engle, R. A., Smith, M., \& Hughes, E. K. (2008). Orchestrating productive mathematical discussions: Five practices for helping teachers move beyond show and tell. Mathematical Thinking and Learning, 10, 313-340. 